$\mathbb{T}$ periodica polytechnica

Transportation Engineering

36/1-2 (2008) 9397

doi: 10.3311/pp.tr.2008-1-2.17

web: http://www.pp.bme.hu/tr

(c) Periodica Polytechnica 2008

RESEARCH ARTICLE

\section{The design of a chassis system based on multi-objective qLPV control}

Charles Poussot-Vassal / Olivier Sename / Luc Dugard / Péter Gáspár / Zoltán

Szabó / József Bokor

Received 2007-03-03

\begin{abstract}
In this paper we compare LTI and $q L P V \mathcal{H}_{\infty} / \mathcal{H}_{2}$ controllers. The Pareto limit is used to show the compromise that has to be done when a mixed synthesis is achieved. Simulations on a nonlinear half vehicle model, with multiple objectives, are performed to show the efficiency of the method.
\end{abstract}

\section{Keywords}

robust control $\cdot$ LPV controlm suspension system · linear matrix inequality (LMI)

\section{Acknowledgement}

This work was supported by the Hungarian National Science Foundation (OTKA) under the grant $T$ - 048482 which are gratefully acknowledged.

\section{Charles Poussot-Vassal}

Olivier Sename

Luc Dugard

Laboratoire d'Automatique de Grenoble, GIPSA-Lab, Grenoble, France

\section{Péter Gáspár}

Zoltán Szabó

\section{József Bokor}

Computer and Automation Research Institute, Hungarian Academy of Sciences, Kende u. 13-17, H-1111 Budapest, Hungary

e-mail: gaspar@sztaki.hu

\section{Introduction}

The main role of suspensions is to improve comfort by isolating the vehicle chassis to an uneven ground and to provide a good road holding to ensure passenger safety. Suspension control of quarter vehicle have been widely explored the past few years to improve vertical movements either by applying LQ [6], Skyhook [8], $\mathcal{H}_{\infty}$ control [5, 12], LPV [4] or mixed synthesis [1,11]. Roll dynamic is catched by the half vehicle model and is directly linked to suspension behaviour. Separated synthesis on each suspension can not guarantee global performances. The aim of the mixed $\mathcal{H}_{\infty} / \mathcal{H}_{2}$ control synthesis is to treat the standard $\mathcal{H}_{2}$ and $\mathcal{H}_{\infty}$ optimal control problems as separate problems but in a unified state-space framework. This method yields a compensator that combines the $\mathcal{H}_{2}$ quadratic performance criterion for disturbance rejection with the $\mathcal{H}_{\infty}$ performance criterion for maximum robustness against destabilizing uncertainties. The controller which minimizes the $\mathcal{H}_{2}$ performance index is selected from the suitable $\mathcal{H}_{\infty}$ controllers, thus the desired criteria are met by creating a balance between $\mathcal{H}_{2}$ and $\mathcal{H}_{\infty}$ norms [3].

The mixed qLPV $\mathcal{H}_{\infty} / \mathcal{H}_{2}$ method is proposed here for the design of active suspension system, in which different optimization criteria are applied to guarantee the performance specifications and the nonlinearity of the suspension system. The nonlinearity in the suspension system is caused by the changes in the spring and damping coefficients. It is assumed that the nonlinear dynamics of road vehicles is approximated by LPV (qLPV) models, in which nonlinear terms are hidden with newly defined scheduling variables and they are available from calculated signals. The active suspension based on the LPV model takes the nonlinear dynamics of the system into consideration. Performance limitations according to the importance given between the $\mathcal{H}_{\infty}$ and the $\mathcal{H}_{2}$ criteria is shown with the Pareto limit.

The paper is organized as follows: in Section 2 we introduce a linear and a nonlinear model of the half vehicle. LTI and qLPV polytopic $\mathcal{H}_{\infty} / \mathcal{H}_{2}$ control, based on LMIs, are presented in Section 3. The Pareto limit, applied to the half vehicle provides smart indications in the way to choose $\mathcal{H}_{\infty} / \mathcal{H}_{2}$ attenuation parameters according to the desired performances. In Section 4 
validations are done on the nonlinear model presented.

\section{Vehicle model of suspension systems}

Roll dynamic is the main movement that enters when a driver turns. The half vehicle model involved here is a chassis model that catches vertical and roll dynamics [8] (Fig. 1). It models the left/right vehicle load transfers that appear during a steering situation. The model is composed of two suspensions, each of them modelled by a spring $\left(F_{k_{\{f l, f r\}}}\right)$, a damper $\left(u_{\{f l, f r\}}=F_{c_{\{f l, f r\}}}\right.$, in the passive case) or an actuator $\left(u_{\{f l, f r\}}=u_{\{f l, f r\}_{\mathcal{H}_{\infty} / \mathcal{H}_{2}}}+\right.$ $c_{\{f l, f r\}}$, in the active case) linked to a common suspended mass $\left(m_{s}\right)$ and to a specific unsprung mass $m_{u s_{f l}}$ and $m_{u s_{f r}}$. Tires $k_{t_{f l}}$ and $k_{t_{f r}}$ are linked to the ground and to the unsprung mass $m_{u s_{f l}}$ and $m_{u s_{f r}}$ respectively. The movements taken into account are the vertical displacement of the suspended mass $\left(z_{s}\right)$, the unsprung masses $\left(z_{u s_{\{f l, f r\}}}\right)$, the suspension deflections $\left(z_{d e f_{\{f l, f r\}}}\right)$ and the roll angle $(\theta)$ of the center of gravity of the suspended mass.

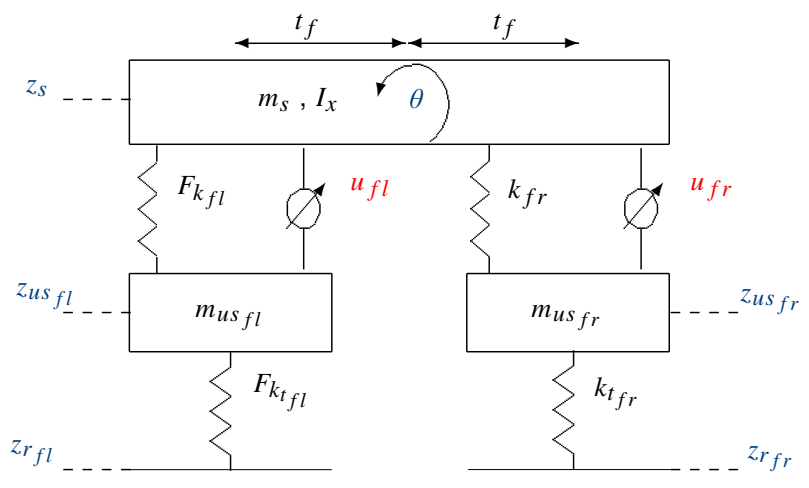

Fig. 1. Half vehicle model

The model is obtained by simply adding two suspensions and tires equations with the dynamical equation of the chassis as follows. First we derive the suspension $\left(F_{s z_{i}}\right)$ and tire $\left(F_{t z_{i}}\right)$ forces,

$$
\left\{\begin{array}{l}
F_{t z_{f l}}=k_{t_{f l}}\left(z_{u s_{f l}}-z_{r_{f l}}\right) \\
F_{t z_{f r}}=k_{t_{f r}}\left(z_{u s_{f r}}-z_{r_{f r}}\right) \\
F_{s z_{f l}}=F_{k_{f l}}\left(z_{s_{f l}}-z_{u s_{f l}}\right)-\frac{k_{b} \theta}{2 l_{f l}}+u_{f l} \\
F_{s z_{f r}}=F_{k_{f r}}\left(z_{s f r}-z_{u s_{f r}}\right)+\frac{k_{b} \theta}{2 l_{f r}}+u_{f r}
\end{array}\right.
$$

then the dynamic of the chassis and unsprung masses (bounce and roll) are given by

$$
\left\{\begin{aligned}
m_{s} \ddot{z}_{s} & =-\left(F_{s z_{f l}}+F_{t z_{f r}}+F_{d z}\right) \\
m_{u s_{f l}} \ddot{z}_{u s_{f l}} & =F_{s z_{f l}}-F_{t z_{f l}} \\
m_{u s_{f r}} \ddot{z}_{u s_{f r}} & =F_{s z_{f r}}-F_{t z_{f r}} \\
I_{x} \ddot{\theta} & =F_{s z_{f l}} t_{f}-F_{t z_{f r}} t_{f}+M_{d x}
\end{aligned}\right.
$$

where $F_{k_{i}}$ and $F_{c_{i}},\{i=f l, f r\}$, represent the force delivered by the spring and by the damper (either linear or nonlinear), $k_{t_{i}}$ is the stiffness of the tire and $k_{b}$ models the influence of an anti-roll bar. $I_{x}$ is the chassis inertia on the roll axis, $t_{f}$ is the distances of the unsprung masses to the center of gravity of the suspended mass. Finally, $\theta, z_{s}, z_{u s_{f l}}$ and $z_{u s_{f r}}$ represent the roll angle and the chassis, unsprung mass left and right bounce. Then $z_{r_{f l}}$ and $z_{r_{f r}}$ represent the road disturbances on the wheels. $F_{d z}, M_{d x}$ represent the load and inertia disturbances. Note that when the passive system is considered, $u_{f r}=F_{c_{f r}}$ and $u_{f l}=F_{c_{f l}}$.

Then, the state space vector of the linear model is defined by

$$
x=\left[\begin{array}{llllllll}
z_{u s_{f l} l} & \dot{z}_{u s_{f l} l} & z_{u s_{f r}} & \dot{z}_{u s_{f r}} & z_{s} & \dot{z}_{s} & \theta & \dot{\theta}
\end{array}\right],
$$

the input are given by $w=$ $\left[\begin{array}{llllll}z_{r_{f l}} & z_{r_{f r}} & F_{d z} & M_{d x} & u_{f l} & u_{f r}\end{array}\right]$ and the measured signal used for control $y=\left[\begin{array}{ll}z_{d e f_{f l}} & z_{d e f_{f r}}\end{array}\right]$.

\section{Mixed $\mathcal{H}_{\infty} / \mathcal{H}_{2}$ LMI based synthesis}

\subsection{A LTI multi-objective controller}

The multi-objective synthesis consists of giving different kinds of constraints on the output of a system. With this formulation (for the case of $\mathcal{H}_{\infty} / \mathcal{H}_{2}$ ), let describe the system as follows

$$
\left[\begin{array}{c}
\dot{x} \\
z_{\infty} \\
z_{2} \\
y
\end{array}\right]=\left[\begin{array}{cccc}
A & B_{\infty} & B_{2} & B \\
C_{\infty} & D_{\infty 1} & D_{\infty 2} & E_{\infty} \\
C_{2} & D_{21} & D_{22} & E_{2} \\
C & F_{\infty} & F_{2} & 0
\end{array}\right]\left[\begin{array}{c}
x \\
w_{\infty} \\
w_{2} \\
u
\end{array}\right]
$$

the controller,

$$
\left[\begin{array}{c}
\dot{x}_{c} \\
u
\end{array}\right]=\left[\begin{array}{ll}
A_{c} & B_{c} \\
C_{c} & D_{c}
\end{array}\right]\left[\begin{array}{l}
x \\
y
\end{array}\right]=S
$$

and the closed loop,

$$
\left[\begin{array}{c}
\dot{x} \\
z_{\infty} \\
z_{2}
\end{array}\right]=\left[\begin{array}{ccc}
\mathcal{A} & \mathcal{B}_{\infty} & \mathcal{B}_{2} \\
\mathcal{C}_{\infty} & \mathcal{D}_{\infty 1} & \mathcal{D}_{\infty 2} \\
\mathcal{C}_{2} & \mathcal{D}_{21} & 0
\end{array}\right]\left[\begin{array}{c}
x \\
w_{\infty} \\
w_{2}
\end{array}\right]
$$

The $\mathcal{H}_{\infty} / \mathcal{H}_{2}$ synthesis consists of, imposing $T_{\infty}=$ $\left\|z_{\infty} / w_{\infty}\right\|_{\infty}<\gamma_{\infty}$ and $T_{2}=\left\|z_{2} / w_{2}\right\|_{2}<\gamma_{2}$. Hence the LMI based problem formulation is the following: minimize $\gamma_{2}$ and $\gamma_{\infty}$ subject to $K$ and $Z$. [2,9]

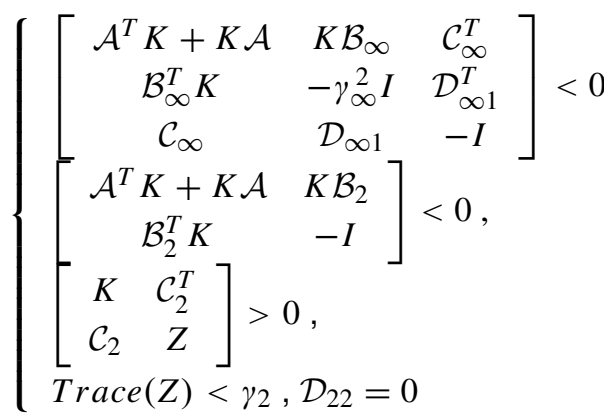

Then solving this problem gives the LTI controller that achieves the desired performances. Note that to relax BMIs (5) into LMIs we use the transformation given in [9].

\subsection{A qLPV multi-objective controller}

Linear parameter varying theory is useful to tackle measurable and bounded nonlinearities. We talk about qLPV when the 
varying parameters only enter in the dynamic matrix $A$ of the system. In the suspension system, the measure of the deflection (used as a controller input) can also be used to reconstruct the stiffness coefficient [12]. To build a qLPV controller, we use the polytopic approach which consists of building a controller to the $k$-corners of the polytope (formed by all the possible combinations of the upper and lower bounds of each varying parameters) and to schedule these $k$-controllers by the measure of the varying variables. The qLPV system is described as follows, with $p$ a varying parameter,

$$
\left[\begin{array}{c}
\dot{x} \\
z_{\infty} \\
z_{2} \\
y
\end{array}\right]=\left[\begin{array}{cccc}
A(p) & B_{\infty} & B_{2} & B \\
C_{\infty} & D_{\infty 1} & D_{\infty 2} & E_{\infty} \\
C_{2} & D_{21} & D_{22} & E_{2} \\
C & F_{\infty} & F_{2} & 0
\end{array}\right]\left[\begin{array}{c}
x \\
w_{\infty} \\
w_{2} \\
u
\end{array}\right]
$$

the parameter dependent controller,

$$
\left[\begin{array}{c}
\dot{x}_{c} \\
u
\end{array}\right]=\left[\begin{array}{ll}
A_{c}(p) & B_{c}(p) \\
C_{c}(p) & D_{c}(p)
\end{array}\right]\left[\begin{array}{c}
x_{c} \\
y
\end{array}\right]=S(p)
$$

and the parameter dependent closed loop,

$$
\left[\begin{array}{c}
\dot{x} \\
z_{\infty} \\
z_{2}
\end{array}\right]=\left[\begin{array}{ccc}
\mathcal{A}(p) & \mathcal{B}_{\infty} & \mathcal{B}_{2} \\
\mathcal{C}_{\infty} & \mathcal{D}_{\infty 1} & \mathcal{D}_{\infty 2} \\
\mathcal{C}_{2} & \mathcal{D}_{21} & 0
\end{array}\right]\left[\begin{array}{c}
x \\
w_{\infty} \\
w_{2}
\end{array}\right]
$$

Then the corresponding mixed problem is similar to the LTI one: minimize $\gamma_{2}$ and $\gamma_{\infty}$ subject to $K$ and $Z$.

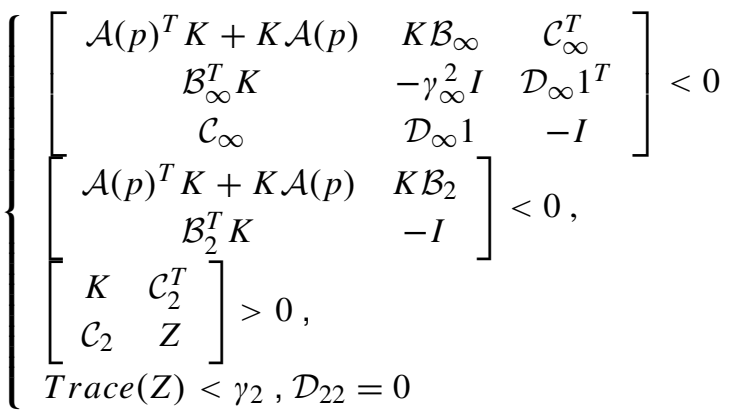

3.3 Design characteristics and performances on the half vehicle model

In the case of a half vehicle, the measure is the suspension deflection and the selected varying variables are the stiffness of the suspension spring, i.e. $k_{f l}$ and $k_{f r}$. The associated polytope is then formed by $k=4$ corners $(10)$ and $k$-controllers.

$$
\Theta=\left[\begin{array}{ll}
\frac{k_{f l}}{k_{f l}} & \overline{k_{f r}} \\
\overline{\overline{k_{f r}}} & k_{f r} \\
\overline{k_{f l}} & \overline{\overline{k_{f r}}}
\end{array}\right], \quad \begin{gathered}
k_{f l} \in\left[\underline{k}_{f l}, \bar{k}_{f l}\right] \\
k_{f r} \in\left[\underline{k}_{f r}, \bar{k}_{f r}\right]
\end{gathered}
$$

According to the dissipative theory, each constraint can be expressed as a supply function, then translated into an LMI 5. 9] [9, 10].

- The $\mathcal{H}_{\infty}$ performance is used to enforce robustness to model uncertainties and to express frequency-domain performance specifications
- The $\mathcal{H}_{2}$ performance can be used to minimize energy of the signal (note the equivalence of these norms in the frequency domain, but not in the time one)

Hence, coupled together, these specifications should improve the single $\mathcal{H}_{\infty}$ constraint. On a half vehicle model the performances we want to reach are multiple. As exposed in [7, 8], some frequency specifications have to be specified concerning the suspension deflection, suspended mass and the unsprung masses (to reduce gain around sensitive low frequencies). Also, weight on the control signal prevents actuator saturation. To these frequency specifications, expressed by the $\mathcal{H}_{\infty}$ theory, the addition of $\mathcal{H}_{2}$ constrain is used to minimize energy of time signals.

Then, the resulting generalized plant is (Fig. 2),

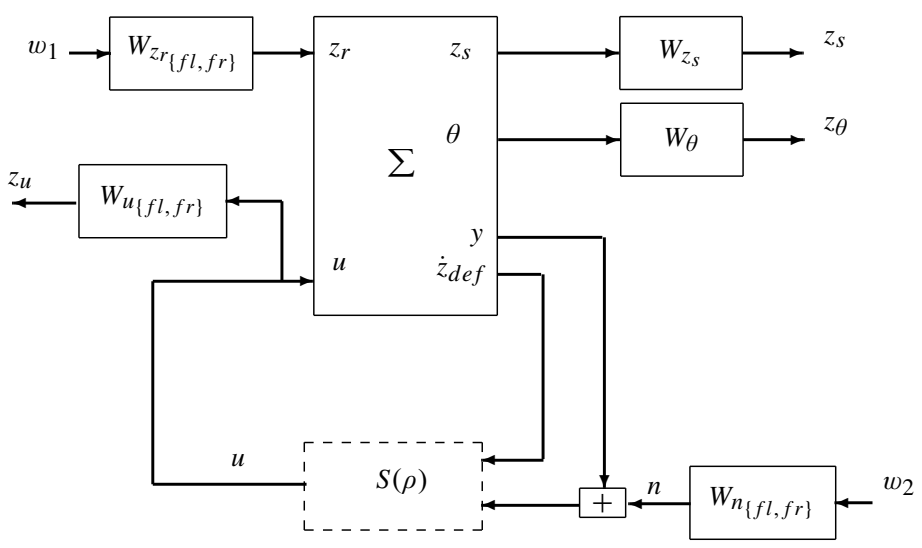

Fig. 2. Generalized plant

In the mixed synthesis the considered $\mathcal{H}_{\infty}$ and $\mathcal{H}_{2}$ controlled output, noted $z_{\infty}$ and $z_{2}$ respectively, are the following:

$$
\begin{aligned}
z_{\infty} & =\left[\begin{array}{llll}
z_{s} & z_{\theta} & z_{u_{f l}} & z_{u_{f l}}
\end{array}\right] \\
z_{2} & =\left[\begin{array}{llll}
z_{s} & z_{\theta} & z_{u_{f l}} & z_{u_{f l}}
\end{array}\right]
\end{aligned}
$$

Note that when we will compare the mixed synthesis to the $\mathcal{H}_{\infty}$ one, the controlled outputs of the $\mathcal{H}_{\infty}$ controller are, $z_{\infty}=$ $\left[\begin{array}{cccc}z_{s} & z_{\theta} & z_{u_{f r}} & z_{u_{f l}}\end{array}\right]$.

\subsection{Pareto limit}

It is impossible to minimize both $\gamma_{\infty}$ and $\gamma_{2}$. In the literature, the mixed problem is generally solved by minimizing a convex combination of $\mathcal{H}_{\infty}$ and $\mathcal{H}_{2}$ that represents a compromise between the two performances. Such a minimization can take the following form,

$$
\begin{gathered}
\min \left\{\alpha_{1} T_{\infty}+\alpha_{2} T_{2}\right\}, \\
\text { where }\left\{\alpha_{1}, \alpha_{2}\right\} \in[0,1] \times[0,1], \alpha_{1}+\alpha_{2}=1
\end{gathered}
$$

Hence a natural problem raises, how to choose in a smart way $\alpha_{1}$ and $\alpha_{2}$. The concept of non-inferiority (also called Pareto optimality) is used here to characterize the objectives. A noninferior solution is one in which an improvement in one objective requires a degradation of an other. In our case the objectives are $\mathcal{H}_{\infty}$ and $\mathcal{H}_{2}$. To plot the Pareto optimum, applied to 
our problem, we iteratively fix the $\gamma_{\infty}$ and minimize the $\gamma_{2}$. The corresponding results are given in Fig. 3 .

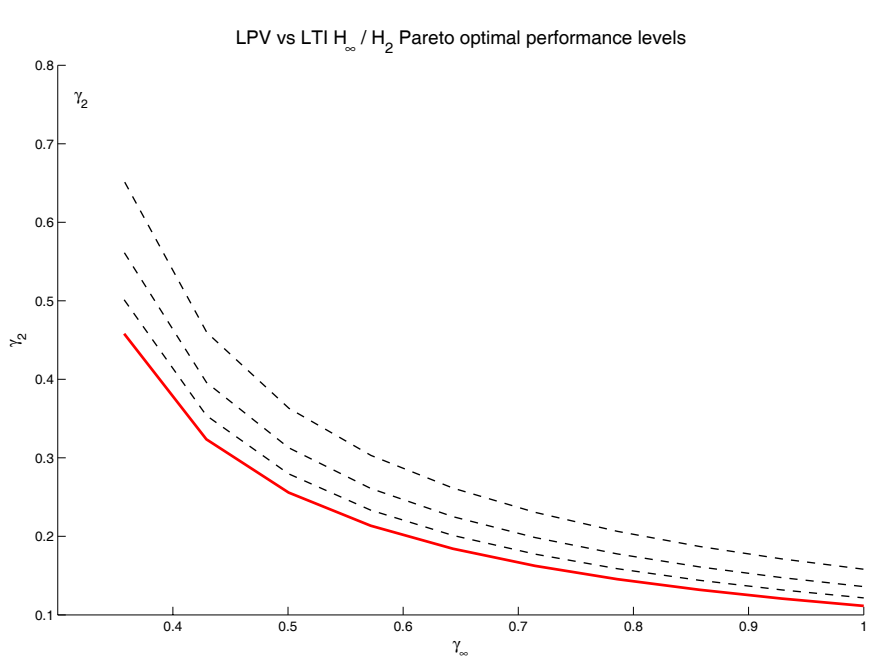

Fig. 3. LTI (solid) vs qLPV (dashed) Pareto limit for $k_{\{f l, f r\}} \in k_{n o m} \times$ $[1,1.2],[1,1.5],[1,2]$

The achievable combinations $\left\{\gamma_{\infty}, \gamma_{2}\right\}$ are the set of couples located over the Pareto limit. The Pareto limit is also useful to measure the conservatism of a method and to exhibit how much one can decrease the performances with a qLPV approach compared to the LTI one. Such a Figure can also motivate researches on polytope reduction. In effect, the more you increase the size of your polytope (bounds of the parameters), the farther you go from the LTI Pareto optimum (Fig. 3), and loose performance.

\section{Simulation results}

To validate the control design, first, simulations are done in order to show the advantages of mixed synthesis compared to single $\mathcal{H}_{\infty}$ objective, then, we study the influence of the choice of the couple $\left\{\gamma_{\infty}, \gamma_{2}\right\}$ on the reached performances. Finally, we compare the LTI mixed approach with the qLPV one. Note that on theses simulations, when a control law is considered, the damper is removed so that the considered suspension simulated is a real semi-active one i.e. a spring (nonlinear) plus an active actuator. In such a way we explicitly model the fact that the damper is replaced by the actuator. Such a control also justify the choice of $\left\{k_{f l}, k_{f r}\right\}$ as varying parameters in the qLPV synthesis.

\subsection{The LTI case}

First we show the advantages of the mixed $\mathcal{H}_{\infty} / \mathcal{H}_{2}$ compared to $\mathcal{H}_{\infty}$ synthesis (for the same $\gamma_{\infty}$ ). In this simulation we generate a step road disturbance on the first then on the second wheel, then a roll moment disturbance and we compare controllers performances according to the passive suspension.

By using the mixed synthesis instead of single $\mathcal{H}_{\infty}$, we reduce the roll angle due to the roll energy minimization (Fig. 4).
Then, we compare the performances of the mixed synthesis for different couples $\left\{\gamma_{\infty}, \gamma_{2}\right\}$ (Fig. 5).
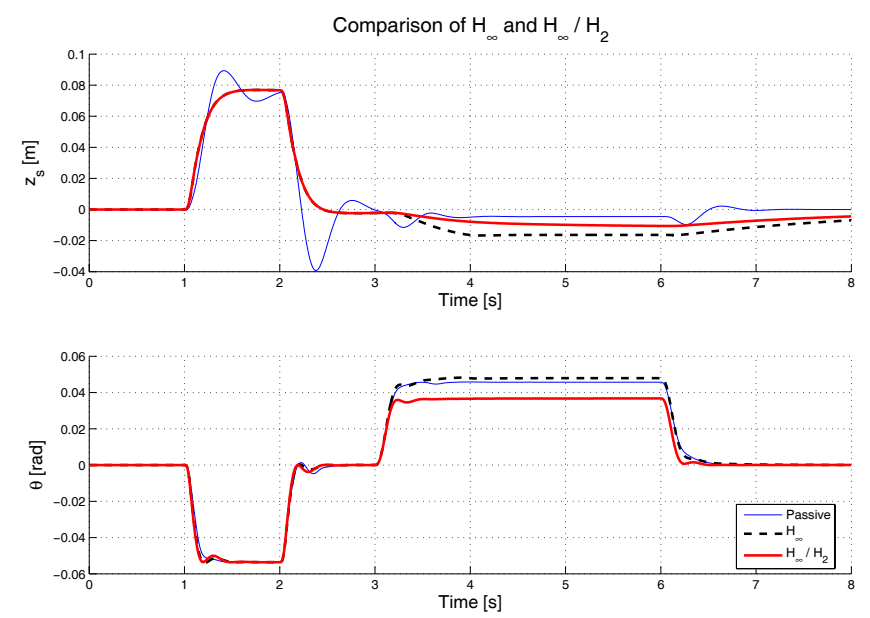

Fig. 4. Comparison between $\mathcal{H}_{\infty}$ (dashed) and Mixed (solid) design with Passive (solid slim)
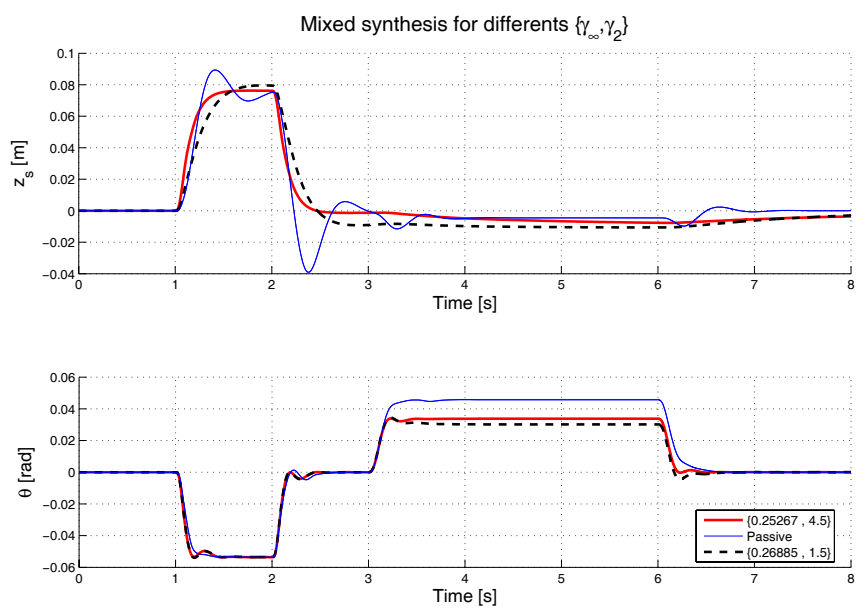

Fig. 5. Comparison of mixed synthesis performances according to different $\left\{\gamma_{\infty}, \gamma_{2}\right\}$

If one decreases the $\gamma_{2}$ attenuation value, then it increases the $\gamma_{\infty}$ one (see Pareto limit Fig. 3). The $\mathcal{H}_{2}$ criteria's aim is to minimize the energy and variations of a signal (here, $z_{s}$ but also the control input are limited). Hence we observe that the $z_{s}$ variations are smoother by using a smaller attenuation gain on the $\mathcal{H}_{2}$ criteria (less oscillations, i.e. ameliorate vertical comfort).

\subsection{The qLPV case}

LTI and the qLPV controllers are here (Figs. 6 and 7) investigated for parameters $k_{\{f l, f r\}}$ varying between $k_{n o m} \times[1,1.95]$ (i.e. $\left.\left[\underline{k}_{f l, f r}, \bar{k}_{f l, f r}\right]\right)$ and for a fixed $\gamma_{\infty}=0.25$. Here, we assume bigger road step disturbance to reach the nonlinear area of the suspension deflection.

The qLPV synthesis improves the performances achieved by the LTI one. Then, such a control tackles the nonlinearities, 

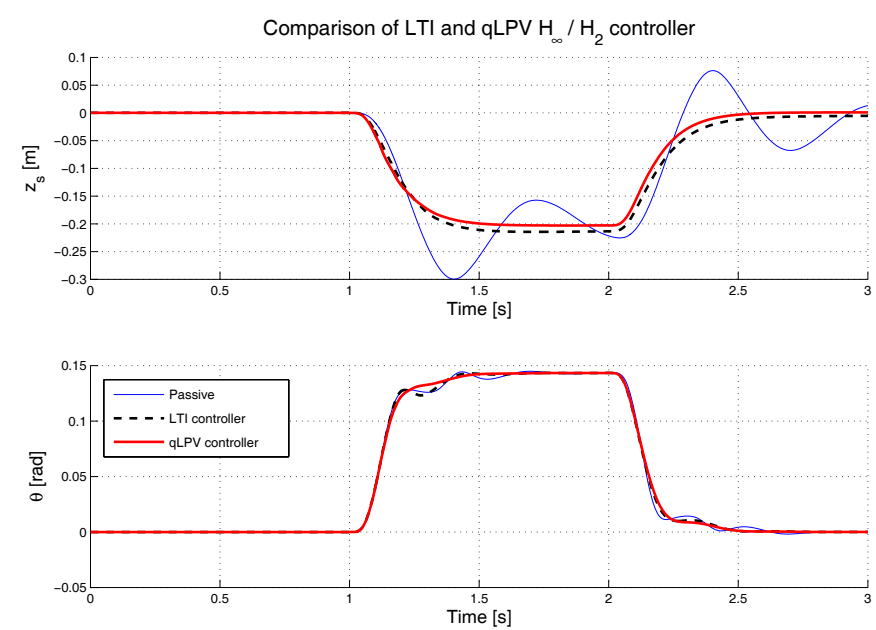

Fig. 6. Comparison of LTI (dashed) and qLPV (solid) mixed synthesis performances
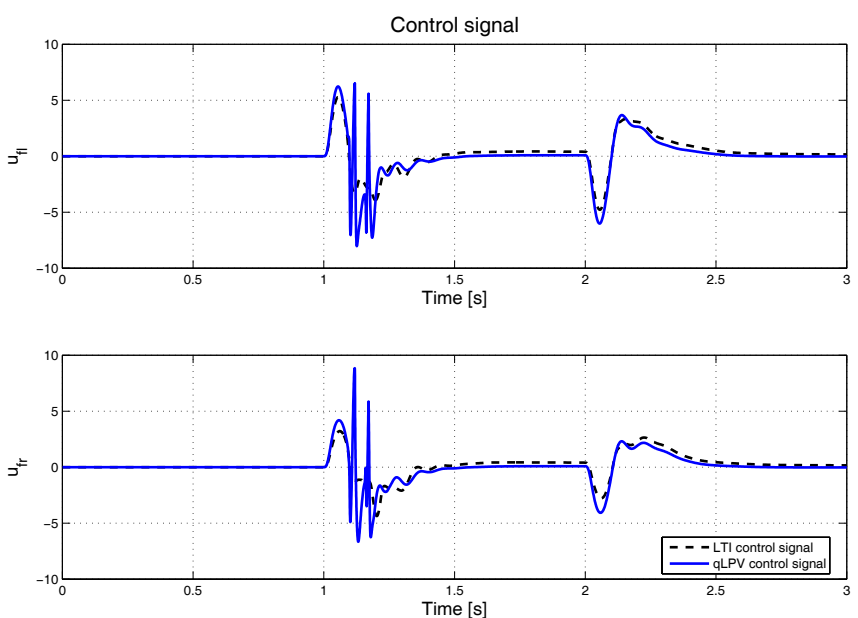

Fig. 7. Control signal $u_{f l}$ (up) and $u_{f r}$ (down) for the LTI (dashed) and qLPV (solid) controller

hence enforces robustness. The $\alpha$ variation shows the scheduling done according to the parameters variations. Note that a qLPV approach, even if enforces robustness, exhibits more complexity than the LTI one because it increases the number of controllers to be synthesized (4 in our case) and requires to schedule them in real-time. Then, the control signal looks sensitive to the parameters variations (Fig.7). Nevertheless, we use in both synthesis (LTI and qLPV) the same number of measures.

\section{Conclusion and future works}

In this paper we investigate a multi-objective mixed qLPV $\mathcal{H}_{\infty} / \mathcal{H}_{2}$ control applied to a half vehicle model. A special interest is made on the advantages of such a synthesis and on the compromises that have to be done in multi-objective applications. By using the Pareto limit (non-inferior solution) we expose a smart way to select the objectives and show the influence on significative driving situations.

\section{References}

1 Abdellahi E, Mehdi D, Saad MM, On the Design of Active Suspension System by $\mathcal{H}_{\infty}$ and mixed $\mathcal{H}_{2} / \mathcal{H}_{\infty}$ : An LMI Approach, Proceedings of the American Control Conference, 2000, pp. 4041-4045.

2 Chilali M, Gahinet $\mathbf{P}, \mathcal{H}_{\infty}$ design with pole placement constraints: an LMI approach, IEEE Transaction on Automatic Control 41 (1996), no. 3, 358-367.

3 Doyle JC, Zhou K, Glover K, Bodenheimer B, Mixed $\mathcal{H}_{2}$ and $\mathcal{H}_{\infty}$ Performance objectives: Optimal Control, IEEE Transaction on Automatic Control 39 (1994), 1575-1587.

4 Fialho I, Balas G, Road adaptive active suspension design using linear parameter varying gain scheduling, IEEE Transaction on Automatic Control 10 (2002), no. 1, 43-54.

5 Gaspar P, Szaszi I, Bokor J, Active Suspension Design Using LPV Control, Proceedings of the 1st IFAC Symposium on Advances in Automotive Control, 2004, pp. 584-589.

6 Hrovat D, Survey of advanced suspension developments and related optimal control application, Automatica 33 (1997), no. 10, 1781-1817.

7 Poussot-Vassal C, Sename O, Dugard L, Ramirez-Mendoza R, Flores L, Optimal skyhook control for semi-active suspensions, Proceedings of the 4th IFAC Symposium on Mechatronics Systems, 2006, pp. 608-613.

8 Sammier D, Sename O, Dugard L, Skyhook and $\mathcal{H}_{\infty}$ control OF ACTIVE VEHICLE SUSPENSIONS: some practical aspects, Vehicle System Dynamics 39 (2003), no. 4, 279-308.

9 Scherer C, Gahinet P, Chilali M, Multiobjective Output-Feedback Control via LMI Optimization, IEEE Transaction on Automatic Control 42 (1997), no. 7, 896-911.

10 Scherer C, Wieland S, LMI in control (lecture support), 2004.

11 Takahashi RHC, Camino JF, Zampieri DE, Peres PLD, A Multiobjective Approach for $\mathcal{H}_{2}$ and $\mathcal{H}_{\infty}$ Active Suspension Control, Proceedings of the American Control Conference, 1998, pp. 48-52.

12 Zin A, Sename O, Gaspar P, Dugard L, Bokor J, An LPV $/ \mathcal{H}_{\infty}$ Active Suspension Control for Global Chassis Technology: Design and Performance Analysis, Proceedings of the IEEE American Control Conference, 2006. 